\title{
A Systematic Mapping Study on Cloud Computing Security
}

\author{
Noman Mazher \\ Department of Information Technology \\ University of Gujrat, \\ Gujrat Pakistan
}

\author{
Imran Ashraf \\ Department of Information Technology \\ University of Gujrat, \\ Gujrat Pakistan
}

\begin{abstract}
From last two decades hype of cloud computing has been increased largely. With all of its advantageous cloud computing has some potential disadvantages. One of which is security .Cloud computing security has been discussed by many researchers. Researchers are paying their much attention about security of cloud computing. In this research we will investigate major areas of research in domain of cloud computing security. By using empirical study method we will statically prove that in which area there is most research in domain of cloud computing security.
\end{abstract}

\section{Keywords}

Cloud computing, cloud computing security, empirical study.

\section{INTRODUCTION}

The world of computation has becoming larger and complex day by day.[1] Could computing is the most popular model for supporting large and complex data. Organizations are moving toward cloud computing for getting benefit of its cost reduction and elasticity features. Yet cloud computing has potential disadvantages and threats. One of the major hurdles in moving to cloud computing is its security and privacy concerns [2-5]. As in cloud computing environment data is out of user possession this increases the risk of data integrity, data confidentiality etc $[6,7]$. Cloud computing security is the area of enormous research yet there has been a little effort in mapping study on cloud computing security issues. Mapping study is actually a method of research that provides a general idea of research areas[8]. Mapping study also helps out to identify type and quantity of research available in that domain. Mapping study should be done before a specific area of research is selected.

Briefly, a mapping study or systematic review (SR) goes through existing primary reports, in-depth reviews and describes their methodology and results[9]. Compared to literature reviews which are common in any research project, a SR has several benefits: a well-defined methodology reduces bias, a wider range of situations and contexts can lower more general conclusions, and use of statistical metaanalysis can detect more than individual studies.

In this paper a mapping study is done for finding out already done research in the area of cloud computing security issues.

In proceeding section a brief introduction of different security models purposed for different security threats is given.

\section{THE SYSTEMATIC MAPPING PROCESS}

This section presents the standard process of systematic mapping study in general and mapping study of research in specific.

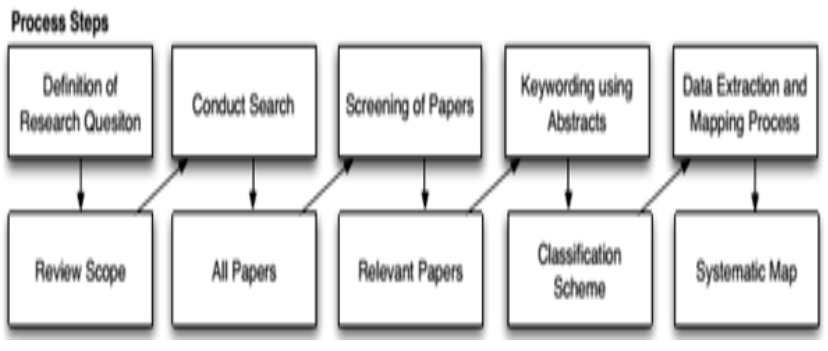

Fig 1 : Systematic mapping study steps[8]

Systematic Mapping study process has the following steps

- Definition of research question

- Conducting search

- $\quad$ Screening of papers

- Keywords

- Data extraction and mapping process

\section{RESEARCH QUESTION:}

Research questions should reflect the main goal of mapping study. They should cover all aspects of defined goals. Research questions of the mapping study are following

RQ 1: Which data sources include papers on cloud computing security?

RQ2: What is the most investigated topic in the field of cloud computing security?

RQ3: In which year most research happened in the area of cloud computing security and in which type of venue they are published?

RQ4: In which type of venue (conference, journal) most papers were published and in which year they were published. 


\section{CONDUCTING SEARCH:}

This is primary phase of mapping study. In this phase first of all search string is designed. This search string is used for searching on different data sources. Search string should be chosen such as it fulfills all prospective of the selected domain. The search string "cloud computing security threats" is used in this mapping study.

\section{Screening of paper for inclusion and exclusion:}

Initially found results in a mapping study are all not mandatory. For refining the search an inclusion and exclusion criteria is used. The following inclusion criteria are used in this mapping study.

Inclusion: books, papers, technical reports and grey literature describing empirical studies regarding object oriented software design. Where several papers reported the same study, only the most recent was included. Where several studies were reported in the same paper, each relevant study was treated separately.

Exclusion: Studies that did not report empirical findings or literature that was only available in the form of abstracts or Powerpoint presentations.

Figure 2: Inclusion exclusion criteria

\section{DATA EXTRACTION AND MAPPING PROCESS}

After inclusion and exclusion process completed data is finalized. On the basis of finalized data the following table was drawn to satisfy the Research Questions.

\begin{tabular}{|l|l|l|l|}
\hline Year & Conference & Journal & Total \\
\hline 2000 & & 1 & 1 \\
\hline 2007 & 2 & & 2 \\
\hline 2008 & & 1 & 1 \\
\hline 2009 & 2 & 1 & 3 \\
\hline 2010 & 1 & 5 & 6 \\
\hline 2011 & 4 & 3 & 7 \\
\hline 2012 & 7 & 2 & 9 \\
\hline 2013 & 4 & 5 & 9 \\
\hline (blank) & & 1 & 1 \\
\hline $\begin{array}{l}\text { Grand } \\
\text { Total }\end{array}$ & 20 & 19 & 39 \\
\hline
\end{tabular}

Fig 3: Year wise and publication wise results

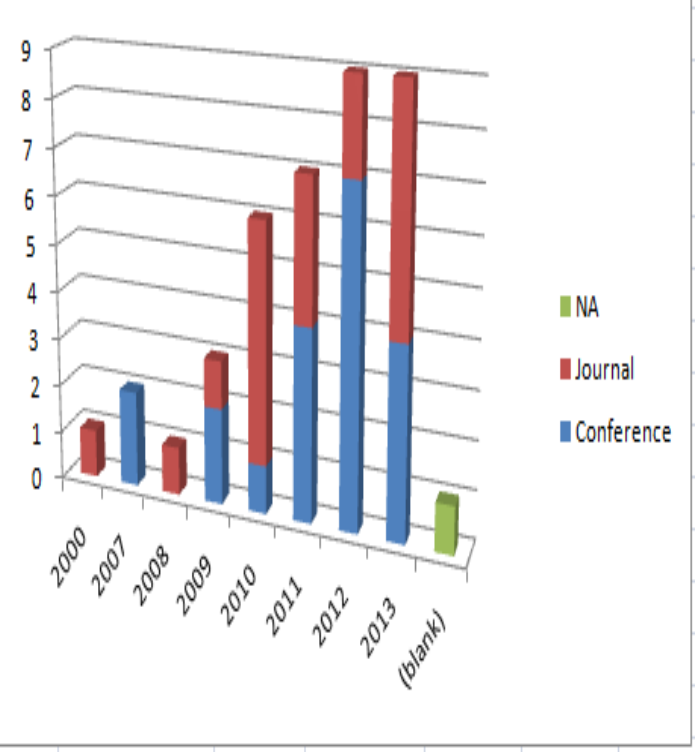

Fig 4: Graph of year and research publication result

\begin{tabular}{lr}
\hline Cloud survey & 3 \\
Insider Threat & 2 \\
PDP & 1 \\
POR & 2 \\
Security model & 9 \\
Security threat survey & 22 \\
Grand Total & 39
\end{tabular}

Fig 5: Research area wise result



Fig 6: Graph of research area wise result 


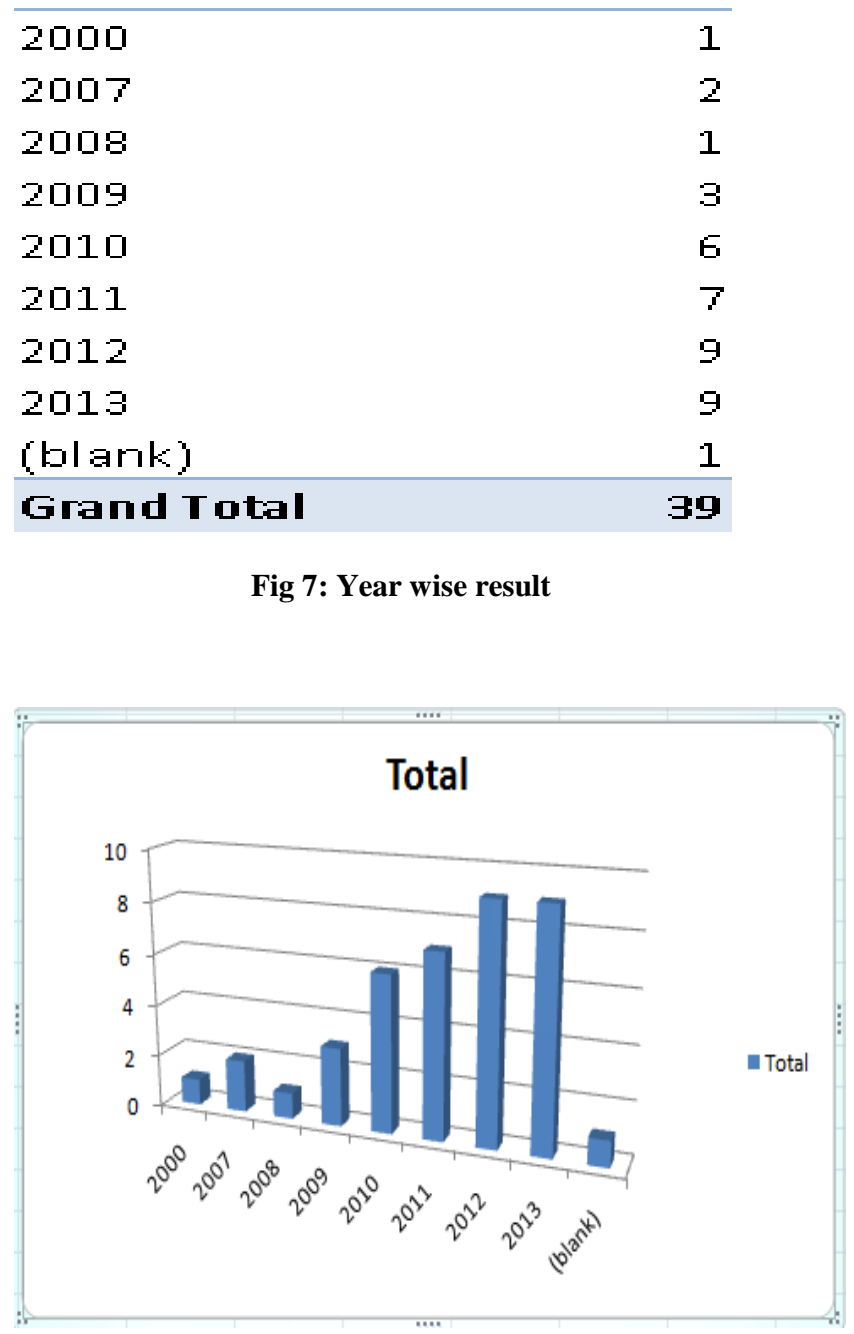

Fig 8: Graph of year wise result

\begin{tabular}{|l|l|}
\hline Institution & Papers \\
\hline ACM & $\mathbf{8}$ \\
\hline Google Scholar & $\mathbf{1 7}$ \\
\hline IEEE & $\mathbf{1 0}$ \\
\hline Springerlink & $\mathbf{4}$ \\
\hline Grand Total & $\mathbf{3 9}$ \\
\hline
\end{tabular}

Fig 9: Search data source

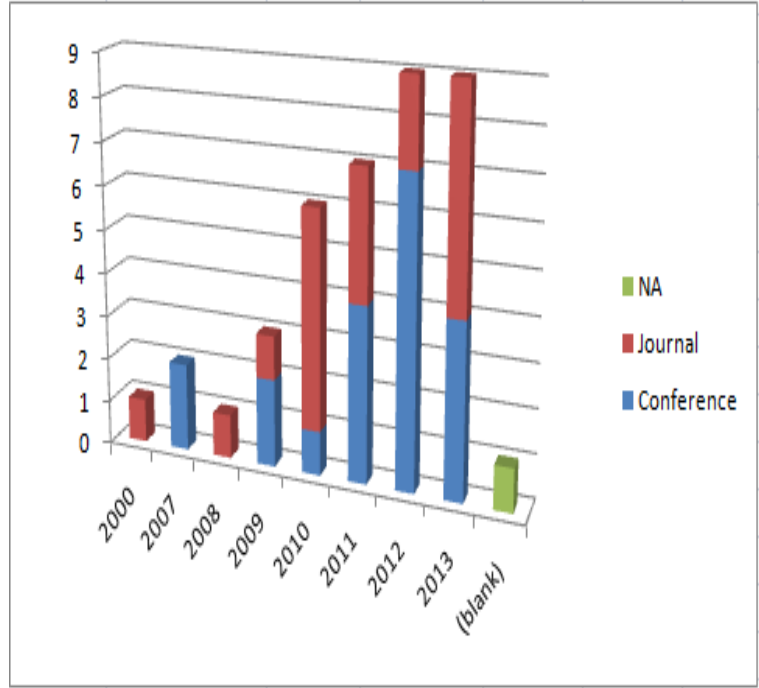

Fig 10 : Graph of research year wise and type of publication wise result

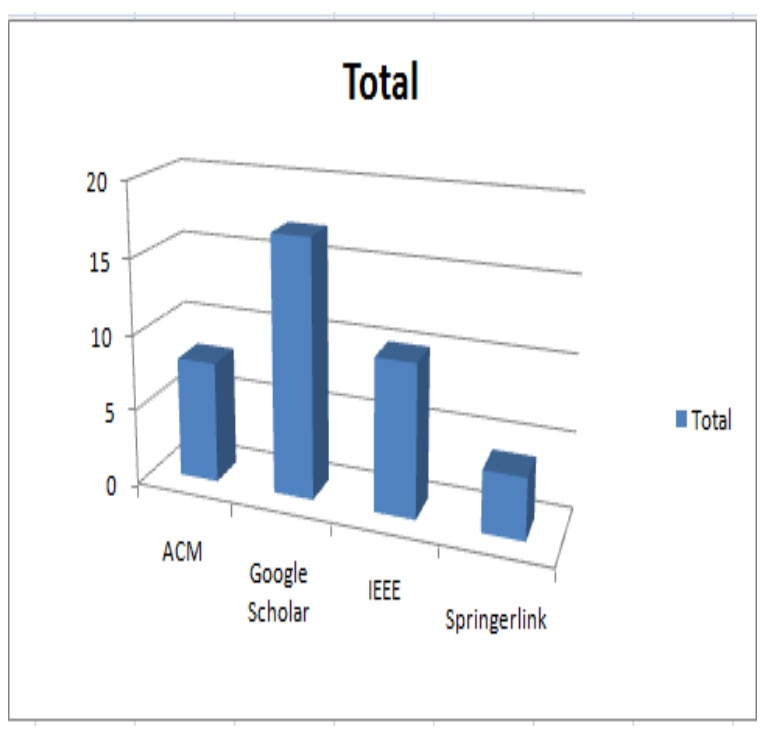

Fig 11: Graph of research engine wise result

\begin{tabular}{|c|c|c|c|c|}
\hline Row Labels - Conference & & Journal & NA & Grand Total \\
\hline 2000 & & 1 & & 1 \\
\hline 2007 & 2 & & & 2 \\
\hline 2008 & & 1 & & 1 \\
\hline 2009 & 2 & 1 & & 3 \\
\hline 2010 & 1 & 5 & & 6 \\
\hline 2011 & 4 & 3 & & 7 \\
\hline 2012 & 7 & 2 & & 9 \\
\hline 2013 & 4 & 5 & & 9 \\
\hline (blank) & & & 1 & 1 \\
\hline Grand Total & 20 & 18 & 1 & 39 \\
\hline
\end{tabular}

Fig 12: Year wise and venue wise result 


\section{CONCLUSION}

This research is based on a mapping study of previous research in the area of cloud computing. The study reveals that the area of cloud computing is very emerging area of research and yet in its infancy. Statistics show that for the last two years this area has been becoming an area of researcher's interest as number of journal and conference publications are increased considerably. Further, security threat area of cloud computing is the most researched area in 2013 which reveals its importance. Results clearly show the emerging importance of cloud computing from future perspective.

\section{REFERENCES}

[1] L. M. Vaquero, L. Rodero-Merino, J. Caceres, and M. Lindner, "A break in the clouds: towards a cloud definition," ACM SIGCOMM Computer Communication Review, vol. 39, pp. 50-55, 12/31/2008 2008 .

[2] S. e. o. c. Pearson and G. e. o. c. Yee, Privacy and security for cloud computing.

[3] D. G. Rosado, Security engineering for cloud computing : approaches and tools.

[4] B. R. Kandukuri, V. R. Paturi, and A. Rakshit, "Cloud security issues," in Services Computing,
2009. SCC'09. IEEE International Conference on, 2009, pp. 517-520.

[5] Y. Chen, V. Paxson, and R. H. Katz, "What's new about cloud computing security," University of California, Berkeley Report No. UCB/EECS-2010-5 January, vol. 20, pp. 2010-5, 2010.

[6] C. Almond, "A practical guide to cloud computing security," A white paper from Accenture and Microsoft, 2009.

[7] H. Takabi, J. B. Joshi, and G.-J. Ahn, "Security and privacy challenges in cloud computing environments," Security \& Privacy, IEEE, vol. 8, pp. 24-31, 2010.

[8] K. Petersen, R. Feldt, S. Mujtaba, and M. Mattsson, "Systematic mapping studies in software engineering," in 12th International Conference on Evaluation and Assessment in Software Engineering, 2008, p. 1.

[9] N. Condori-Fernandez, M. Daneva, K. Sikkel, R. Wieringa, O. Dieste, and O. Pastor, "A systematic mapping study on empirical evaluation of software requirements specifications techniques," in Proceedings of the 2009 3rd International Symposium on Empirical Software Engineering and Measurement, 2009, pp. 502-505. 